\title{
MODULI FOR ANALYTIC LEFT ALGEBRAIC GROUPS
}

BY

\author{
ANDY R. MAGID
}

\begin{abstract}
ABSTRACr. This paper classifies left algebraic group structures on faithfully representable complex analytic groups by establishing the existence of an algebraic variety whose complex points correspond to such structures on a given analytic group.
\end{abstract}

Let $G$ be a faithfully representable complex analytic group. An analytic left algebraic group structure on $G$ [7, Definition 2.1, p. 1052] is a finitely generated C-subalgebra $A$ of the algebra $R$ generated by all the matrix coordinate functions of finite-dimensional analytic representations of $G$, such that $A$ is stable under right translations by elements of $G$, and such that the maximal ideal space of $A$ is bijective with $G$ ( $g$ in $G$ corresponds to the kernel of the algebra homomorphism given by evaluation at $g)$. Thus the pair $(G, A)$ is an affine variety over $\mathbf{C}$ such that left translations by elements of $G$ are morphisms. The structure is normal basic if $G$ is a semidirect product as analytic left algebraic groups of a maximal simply-connected solvable normal subgroup of $G$ (a nucleus of $G$ ) and a maximal reductive subgroup of $G$. (These structures were introduced and studied by Hochschild and Mostow [2].)

The purpose of this paper is to classify left algebraic group structures by establishing the existence of a moduli space for the normal basic left algebraic group structures on a given group $G$. This construction includes constructing a moduli space for the nuclei of $G$, and this latter space is explicitly determined.

The construction proceeds by translating the problem to that of constructing moduli for certain subalgebras of the Lie algebra of $G, L(G)$. The core of an analytic left algebraic group structure $A$ on $G$ is the set $C(G)$ of all elements $g$ in $G$ such that right translation by $g$ is also a morphism of the variety $(G, A)[7$, Definition 1.2, p. 1047]. Then $C(G)$ is an algebraic subgroup of $G$ [7, Theorem 1.7, p. 1048] and its unipotent radical $U$ is such that if $K$ is the associated nucleus of $G$ then $L(U)$ is a Cartan subalgebra of $L(K)$ [8, Corollary 1.9, p. 172]. Moreover, the (analytic) subgroup $U$ of $G$ determines the left algebraic group structure $A$ [8, Theorem 2.3, p. 174], and every Cartan subalgebra of $L(K)$ arises in this fashion ([8, Theorem 1.10, p. 172] and (0.1) below). It follows that a moduli space for the Cartan subalgebras of the Lie algebras of nuclei of $G$ is a moduli space for the normal basic left algebraic group structures on $G$, and this space is constructed as a subspace of the moduli space for subalgebra bundles of Lie algebra bundles on a variety [1, Theorem 2.2 , p. 62].

Received by the editors July 11, 1977 and, in revised form, November 14, 1979.

AMS (MOS) subject classifications (1970). Primary 22E10, 22 E20. 


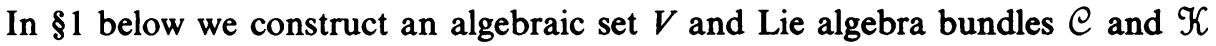
on $V$ such that points of $V$ correspond to pairs $(C, K)$, where $K$ is the Lie algebra of a nucleus of $G$ and $C$ is a Cartan subalgebra of $K$; the correspondence sends $x$ in $V$ to the pair of fibres $(\mathcal{C}(x), \mathcal{K}(x))$. This construction proceeds by first constructing an algebraic set $W$ and a Lie algebra bundle $\mathscr{H}$ on $W$ such that points of $W$ correspond to Lie algebras of nuclei of $G$ under the correspondence which sends $x$ to the fibre $\mathcal{H}(x)$, then stratifying $W$ by the subvarieties of points corresponding to Lie algebras of nuclei with Cartan subalgebras of given dimension, and then constructing the piece of $V$ over each of these subvarieties.

In $\$ 2$, we explicitly describe $W$ as the linear variety $\operatorname{Hom}_{\mathbf{C}}(\mathfrak{f} /[\mathfrak{g}, \mathfrak{h}], \mathfrak{h})$ where $\mathfrak{g}=L(G), \mathfrak{f}$ is the Lie algebra of a nucleus of $G$ and $\mathfrak{h}$ is the Lie algebra of a maximal reductive subgroup of $G$. The nilpotent nuclei correspond to algebraic group structures on $G$. We show that this set is the linear subvariety $\operatorname{Hom}_{\mathbf{C}}\left(\mathfrak{f} /[\mathfrak{g}, \mathfrak{f}], \mathfrak{h}^{\mathfrak{l}}\right)$ of $W$, where $\mathfrak{f}$ is nilpotent and $\mathfrak{h}^{\mathfrak{l}}$ is the centralizer of $\mathfrak{f}$ in $\mathfrak{h}$. Hochschild and Mostow have also identified this set with a linear variety [2, Theorem 8.6, p. 135], and we show that the varieties are isomorphic.

In $\$ 3$ we construct an algebraic cross section to the projection from $V$ to $W$. Its image then provides a set of representatives for the $G$-conjugacy classes of left algebraic group structures on $G$.

The definitions used above will be employed throughout. All affine algebras, Lie algebras, and algebraic sets are over C. A faithfully representable complex analytic group will be called an FR group. The Cartan rank of a Lie algebra is the dimension of a Cartan subalgebra. The basic reference for nuclei of FR groups is [2], and the references for analytic left algebraic groups are [3], [6], [7], and [8].

The following lemma makes explicit a correspondence developed in [8].

Lemma 0.1. Let $G$ be an $F R$ group and $K$ a nucleus of $G$. The normal basic left algebraic group structures on $G$ with nucleus $K$ correspond one-one with the Cartan subalgebras of $L(K)$.

Proof. Let $H$ be a maximal reductive subgroup of $G$. By [8, §2], there is a Cartan subalgebra of $L(K)$ normalized by $L(H)$. The conjugacy of Cartan subalgebras then implies that every Cartan subalgebra $C$ of $L(K)$ is normalized by the Lie algebra of some maximal reductive subgroup of $G$. By [8, Theorem 1.10, p. 172] there is a normal basic left algebraic group structure on $G$ such that the Lie algebra of the unipotent radical of the core of this structure is $C$. By [8, Lemma 2.5, p. 176] this unipotent radical determines the core and by [8, Theorem 2.3, p. 174] the core determines the left algebraic group structure.

1. The space of left algebraic group structures. In this section we establish the existence of the desired moduli spaces for left algebraic group structures and nuclei. We begin by determining the Lie algebras of nuclei.

LEMMA 1.1. Let $G$ be an FR group and $H$ a maximal reductive subgroup of $G$. The nuclei of $G$ correspond one-one with the ideals of $L(G)$ which are direct sum complements to $L(H)$, the nucleus $K$ corresponding to the ideal $L(K)$. 
Proof. Let $\mathfrak{g}=L(G)$ and $\mathfrak{h}=L(H)$. If $K$ is a nucleus of $G, L(K)$ is an ideal of $g$ of the desired type. Conversely, let $\mathfrak{f}$ be an ideal of $g$ which is a direct sum complement to $\mathfrak{h}$.

We first show that is solvable. Let $K^{\prime}$ be any nucleus of $G$ and let $\mathfrak{f}^{\prime}=L\left(K^{\prime}\right)$. Then $\mathfrak{f}^{\prime}$ is solvable and $\operatorname{rad}(\mathfrak{g})=\operatorname{rad}(\mathfrak{h})+\mathfrak{l}^{\prime}$. But also $\mathfrak{g} /(\operatorname{rad}(\mathfrak{h})+\mathfrak{f})=\mathfrak{h} / \operatorname{rad}(\mathfrak{h})$ is semisimple, so $\operatorname{rad}(\mathfrak{h})+\mathfrak{f}$ contains $\operatorname{rad}(\mathfrak{g})=\operatorname{rad}(\mathfrak{h})+\mathfrak{f}^{\prime}$. Since $\operatorname{dim}(\mathfrak{f})=\operatorname{dim}\left(\mathfrak{f}^{\prime}\right)$ and $\operatorname{rad}(\mathfrak{h}) \cap \mathfrak{t}=0$, the containment is equality. Thus $\mathfrak{t}$ is contained in $\operatorname{rad}(\mathfrak{g})$ and hence is solvable.

By [2, Lemma 2.1, p. 113], we can write $f=g_{1}+g_{2}$, where $g_{1}$ is the nilpotent ideal $[\mathfrak{g}, \mathfrak{f}]$ of $\mathfrak{g}$ and $\mathfrak{z}_{2}$ is a nilpotent subalgebra of $\mathfrak{f}$ with $\left[\mathfrak{h}, \mathfrak{g}_{2}\right]=0$. Using the fact that $\operatorname{rad}(\mathfrak{g})=\mathfrak{f}+\operatorname{rad}(\mathfrak{h})$, and that $\mathfrak{f}$ is an ideal, we find that $\mathfrak{z}_{1}=[\operatorname{rad}(\mathfrak{g}), \mathfrak{g}]$ is independent of $f$. Let $K_{i}=\exp \left(\mathcal{S}_{i}\right)$ be the analytic subgroup of $G$ corresponding to $\mathfrak{\xi}_{i}$. Since $\mathfrak{z}_{1}=\left[\mathfrak{f}^{\prime}, \mathfrak{g}\right], K_{1}$ is a closed simply connected normal subgroup of $G$ contained in $K^{\prime}$. Now $K_{2}$ is faithfully representable, so $K_{2}=U \cdot T$ where $U$ is a nucleus of $K_{2}$ and $T$ is a maximal reductive subgroup of $K_{2}$. Since $\left[\mathfrak{h}, \mathfrak{g}_{2}\right]=0, H$ centralizes $T$, so $T H$ is a reductive subgroup of $G$ and $T H=H$ by maximality. Since $L(T) \cap L(H)=0, T=\{e\}$. Thus $K_{2}$ is simply connected and $K=K_{1} K_{2}$ is a simply connected solvable normal analytic subgroup of $G$ with $L(K)=\mathfrak{f}$. To see that $K$ is a nucleus, we must show that $K$ is closed and $G / K$ is reductive. It will be sufficient to verify these for $K / K_{1}$ in $G / K_{1}$, so we now assume $K_{1}=\{e\}$, i.e. that $\mathfrak{g}_{1}=[\operatorname{rad}(\mathrm{g}), \mathrm{g}]=0$. This implies that the radical $R$ of $G$ is central, hence abelian, so $R=K^{\prime} \times T$ where the nucleus $K^{\prime}$ is now a vector group and $T=\operatorname{rad}(H)$ is a torus. Since $L(R)=L(K) \oplus L(T)=L\left(K^{\prime}\right) \oplus L(T)$, there is a Lie algebra automorphism of $L(R)$ fixing $L(T)$ and sending $L\left(K^{\prime}\right)$ to $L(K)$. This induces an automorphism of $R$ sending $K^{\prime}$ to $K$, so $K$ is closed in $G$, and $R / K$ is isomorphic to $T$. Thus $G / K$ has a torus radical, so is reductive.

Because of Lemma (1.1), we can identify the set of nuclei of an FR group with a set of ideals of its Lie algebra.

Definition 1.2. Let $L$ be a Lie algebra and $S$ a subalgebra of $L$. Then $N(S, L)$ denotes the set of ideals $I$ of $L$ with $I \oplus S=L$. If $G$ is an FR group and $H$ is a maximal reductive subgroup of $G$, then $\operatorname{Nuc}(G)=N(L(H), L(G))$.

By Lemma (1.1), $\operatorname{Nuc}(G)$ is a one-one correspondence with the set of nuclei of $G$. We now see that $\operatorname{Nuc}(G)$ carries a natural structure of affine algebraic set.

TheOrem 1.3. Let $L$ be a Lie algebra and $S$ a subalgebra of $L$. Then $N(S, L)$ carries the structure of an affine algebraic set. Moreover, there is a subalgebra bundle $g$ of the Lie algebra bundle $N(S, L) \times L$ such that for each geometric point $x$ of $N(S, L)$ the corresponding ideal of $L$ is $G(x)$.

Proof. Let $r=\operatorname{dim}(L)-\operatorname{dim}(S)$ and let $Z$ be the projective variety $\operatorname{Lie}_{L}^{r}[1$, Theorem 2.2iii, p. 62] which parametrizes the Lie subalgebras of $L$ of dimension $r$, let $\mathcal{H}=\mathcal{K}_{L}$ be the universal bundle over $Z$ of Lie subalgebras of $Z \times L$ of dimension $r$ [1, Theorem 2.2ii, p. 62] and let $W=\{z \in Z \mid \mathcal{H}(z)$ is an ideal of $L$ complementary to $S\}$. We need to show that $W$ is an affine algebraic set.

Let $F=\{z \in Z \mid \mathcal{H C}(z)$ is an ideal of $L\}$. If $\mathcal{E}$ is the constant sheaf of sections of 
$Z \times L$, and $\mathbb{Q}$ is the coherent sheaf $\mathcal{L} /(\mathcal{H}+[\mathcal{L}, \mathcal{H}])$, then $F=\left\{z \in Z \mid \operatorname{rank}\left(\mathbb{Q}_{z}\right)\right.$ $<\operatorname{dim}(S)\}$ so $F$ is closed in $Z$.

Let $G(r, L)$ denote the Grassmann variety of $r$ planes in $L$. Then the set of $r$-planes in $L$ which are not direct sum complements to $S$ is the codimension one Schubert cell in $G(r, L)$. That is, it is a hyperplane section, so its complement $U$ is affine. Since $W=F \cap U$ is closed in $U, W$ is also affine. Take $\mathscr{G}=\mathcal{H} \mid W$.

COROLlary 1.4. Let $G$ be an FR group. Then $\operatorname{Nuc}(G)$ carries a structure of affine algebraic set, and there is a subalgebra bundle $\mathcal{K}$ of the Lie algebra bundle $\operatorname{Nuc}(G) \times L(G)$ such that for each geometric point $x$ of $\mathrm{Nuc}(G)$ the corresponding ideal of $L(G)$ is $\mathscr{K}(x)$.

As explained above, the normal basic left algebraic group structures on an FR group $G$ correspond to Cartan subalgebras of the elements of $\operatorname{Nuc}(G)$. In order to algebraically parametrize these structures, we need to stratify $\operatorname{Nuc}(G)$ by Cartan rank. This requires some facts about linear algebra over commutative rings.

REMARK 1.5. Let $R$ be a commutative ring, $V$ a finitely generated projective $R$-module of constant rank $n$ and $T$ an $R$-endomorphism of $V$. Then $\operatorname{char}(T, R, X)$ $=\sum_{i=0}^{n} c_{i}(T, R) X^{i}$ in $R[X]$ is the characteristic polynomial of $T$ in the sense of [5, p. 108]. Suppose that $\operatorname{char}(T, R, X)$ factors as $X^{s} g$, where the constant term of $g$ is a unit. Let $V_{0}=\operatorname{Ker}(g(T))$ and $V_{1}=\operatorname{Ker}\left(T^{s}\right)$. Then $g$ and $X^{s}$ generate the unit ideal of $R[X]$, so $V=V_{0} \oplus V_{1}$ and $T\left(V_{i}\right) \subseteq V_{i}$. For any commutative $R$-algebra $S, T \otimes S$ is nilpotent on $V_{0} \otimes S$ and $T \otimes S \mid V_{1} \otimes S$ is an isomorphism, so $V_{0} \otimes S=\{x \in V \otimes S \mid T \otimes S$ is nilpotent on $x\}$. If $m$ is a maximal ideal of $R$ and $\bar{R}=R / m$, then $\operatorname{char}(T \otimes \bar{R}, \bar{R}, X)=X^{s} \bar{g}$ where $\bar{g}$ has nonzero constant term, so $\{x \in V \otimes \bar{R} \mid T \otimes \bar{R}$ is nilpotent on $x\}$ has dimension $s$. Thus by the preceding observation (with $S=\bar{R}$ ), we have that $V_{0} \otimes \bar{R}$ has dimension $s$. Thus $V_{0}=\{x \in V \mid T$ is nilpotent on $x\}$ is projective of constant rank $s$.

We now return to Lie algebras. If $L$ is a Lie algebra, and if there is $h$ in $L$ with $c_{i}\left(\operatorname{ad}_{L}(h), C\right) \neq 0$, then the Cartan rank of $L$ does not exceed $i$ [4, Theorem 45, p. 43].

Proposition 1.6. Let $V$ be an algebraic set and let $\mathcal{L}$ be a Lie algebra bundle on $V$. For each integer $j, C_{j}(V, \mathcal{E})=\{v \in V \mid \mathcal{L}(v)$ has Cartan rank at most $j\}$ is open in $V$.

Proof. We may assume that $V$ is affine with coordinate ring $R$ and that $\mathcal{L}$ is trivial as a bundle over $V$. Then $\mathcal{L}$ corresponds to a free $R$-module $L$ with basis, say, $a_{1}, \ldots, a_{n}$. Let $X_{1}, \ldots, X_{n}$ be indeterminates and let $S=R\left[X_{1}, \ldots, X_{n}\right]$. Consider $\operatorname{ad}\left(\Sigma a_{i} X_{i}\right)=T$ as an $S$-endomorphism of the $S$-Lie algebra $S \otimes L$, and let $c_{j}=c_{j}(T, S)$. Now $c_{j}$ is a polynomial function on $V \times \mathbf{C}^{(n)}$, and

$$
U_{j}=\operatorname{pr}_{1}\left(V \times \mathbf{C}^{(n)}-c_{j}^{-1}(0)\right)
$$

is the open subset of all $v$ in $V$ such that there exists some $x=\left(x_{1}, \ldots, x_{n}\right)$ in $\mathbf{C}^{(n)}$ with $0 \neq c_{j}(v, x)=c_{j}\left(\operatorname{ad}\left(\Sigma a_{i}(v) x_{i}\right), \mathbf{C}\right)$, so $U_{j}=C_{j}(V, \mathcal{E})$.

Definition 1.7. Let $G$ be an FR group. Then $\operatorname{Nuc}_{i}(G)=\{I \in \operatorname{Nuc}(G) \mid I$ has Cartan rank $i$ \}. 
COROLlaRY 1.8. Let $G$ be an FR group. Then $\operatorname{Nuc}_{i}(G)$ carries the structure of an open subset of an affine algebraic set and there is a Lie algebra bundle $\mathcal{K}_{i}$ on it such that the geometric point $x$ of $\mathrm{Nuc}_{i}(G)$ corresponds to the ideal $\mathcal{K}_{i}(x)$ of $L(G)$.

Proof. By (1.6), $\operatorname{Nuc}_{i}(G)$ is locally closed in the structure that $\operatorname{Nuc}(G)$ carries from Theorem 1.3, and the bundle on $\operatorname{Nuc}_{i}(G)$ is the restriction of the bundle from Theorem 1.3.

To parametrize those normal basic left algebraic group structures on an FR group with associated nuclei of Cartan rank $i$, we need to produce a universal Cartan subalgebra bundle of the bundle $\mathcal{K}_{i}$ of Corollary 1.8 .

We will need to know that the points where the fibres of a nilpotent subalgebra bundle of a Lie algebra bundle are Cartan form an open set. This is a local question, so we may pass to the affine case.

LEMMA 1.9. Let $R$ be an affine algebra and let $L$ be an $R$-Lie algebra, finitely generated and projective of rank $n$ as an $R$-module. Suppose that for every maximal ideal $m$ of $R, L / m L$ is nilpotent. Then $L$ is nilpotent.

Proof. Let $I$ be the nilradical of $R$. Then the $n$th term of the lower central series of $L, L^{n}$, is contained in $I L=\bigcap\{m L \mid m$ maximal in $R\}$. Then an easy induction shows that $L^{k n} \subseteq I^{k} L$, and since $I$ is nilpotent the right-hand side is zero for large enough $k$.

LEMMA 1.10. Let $R$ be an affine algebra and let $L$ be an $R$-Lie algebra, finitely generated and projective of constant rank as an $R$-module. Suppose that for each maximal ideal $m$ of $R, L / m L$ has Cartan rank $t$. Let $C$ be a subalgebra of $L$ which is an $R$-module direct summand of constant rank $t$ and suppose $C / m C$ is nilpotent for each maximal ideal $m$. If for some maximal ideal $n, C / n C$ is Cartan in $L / n L$, there is an $f$ in $R-n$ such that $C / m C$ is Cartan in $L / m L$ for all maximal $m$ with $f \notin m$.

Proof. Let () denote reduction $\bmod n$. Choose a regular element $x$ of $\bar{L}$ such that $\bar{C}=\bar{L}_{0}(x)=\{z \in \bar{L} \mid \operatorname{ad}(x)$ is nilpotent on $z\}$. Then $\operatorname{char}(\operatorname{ad}(x), \bar{R}, X)=X^{t} g$, where $g(0) \neq 0$. Choose $y \in C$ with $\bar{y}=x$. If $m$ is maximal and $c_{i}(\operatorname{ad}(y) \otimes$ $R / m, R / m) \neq 0$ for $i<t$, then $L / m L$ would have Cartan rank less than $t$, so $c_{i}(\operatorname{ad} y, R)=0$ for $i<t$. Thus $\operatorname{char}(\operatorname{ad} y, R, X)=X^{t} h$ where $\bar{h}=g$. Since $g(0) \neq$ 0 , there is $f \in R-h$ such that $h(0)$ is a unit in $R[1 / f]$. Replace $R$ by $R[1 / f]$. Then, by $\operatorname{Remark} 1.5, L_{0}(y)=\{z \in L \mid \operatorname{ad}(y)$ is nilpotent on $z\}$ is a direct summand of $L$ of constant rank $t$. By Lemma $1.9, C$ is contained in $L_{0}(y)$, and since both are summands of $L$ of rank $t, C=L_{0}(y)$. Now for any maximal $m$, $\operatorname{char}(\operatorname{ad}(y) \otimes R / m, R / m, X)=X^{t}(h \otimes R / m)$ so $y+m$ is regular in $L / m L$, and by Remark $1.5 L_{0}(y) \otimes R / m=\{z \in L / m L \mid \operatorname{ad}(y+m)$ is nilpotent on $z\}$, so $L_{0}(y) \otimes R / m=C / m C$ is Cartan in $L / m L$.

We rephrase Lemma 1.10 geometrically.

COROLlaRY 1.11. Let $V$ be an algebraic set and $\mathcal{L}$ a Lie algebra bundle over $V$ such that $\mathcal{L}(v)$ has Cartan rank $t$ for all $v$ in $V$. Let $\mathcal{C}$ be a subalgebra bundle of $\mathcal{L}$ of rank $t$. Then $\{v \in V \mid \mathcal{C}(v)$ is Cartan in $\mathcal{L}(v)\}$ is locally closed in $V$. 
Proof. By Proposition 1.6, $F=\{v \in V \mid \mathcal{C}(v)$ is nilpotent $\}=\{v \in V \mid \mathcal{C}(v)$ has Cartan rank $t$ \} is locally closed in $V$. Replace $V$ with $F$ and pass to an affine open subset $U$ in $F$. By Lemma 1.10, $\{u \in U \mid \mathcal{C}(u)$ is Cartan in $\mathcal{L}(u)\}$ is open in $U$ and hence in $F$. Thus $\{v \in V \mid \mathcal{C}(v)$ is Cartan in $\mathcal{L}(v)\}$ is open in the closed set $F$, and hence locally closed.

We are now in a position to parametrize normal basic left algebraic group structures on an FR group $G$. By Lemma 0.1, the structures with nucleus $K$ correspond to Cartan subalgebras of $L(K)$. The following definition is motivated by this fact along with Lemma 1.1.

Definition 1.12. Let $G$ be an FR group and $H$ a maximal reductive subgroup. Then $\operatorname{Lag}_{n}(G)$ denotes the set of pairs $(\mathfrak{f}, \mathfrak{c})$, where $\mathfrak{f}$ is an ideal of $L(G)$ of Cartan rank $n$ which is a direct sum complement to $L(H)$, and $c$ is a Cartan subalgebra of f.

TheOREM 1.13. Let $G$ be an FR group. Then, for each integer $n, \operatorname{Lag}_{n}(G)$ carries a structure of algebraic set. Moreover, there are subalgebra bundles $\mathcal{C} \subseteq \mathcal{K}$ of $L(G) \times$ $\operatorname{Lag}_{n}(G)$ such that a geometric point $x$ of $\operatorname{Lag}_{n}(G)$ corresponds to the pair $(\mathscr{K}(x), \mathcal{C}(x))$. The function $\operatorname{Lag}_{n}(G) \rightarrow \mathrm{Nuc}_{n}(G)$, which sends $x$ to the point of $\operatorname{Nuc}_{n}(G)$ corresponding to the ideal $\mathcal{K}(x)$ of $L(G)$, is a surjective morphism.

Proof. Let $Z=\operatorname{Nuc}_{n}(G)$ with the algebraic set structure and Lie algebra bundle $g$ given by Corollary 1.8. Let $W=\mathrm{Lie}_{g / z}^{n}[1$, Theorem 2.7ii, p. 62] and let $G$ be the universal subalgebra bundle of $\mathcal{K}=\mathcal{G} \times_{z} W$. By definition of $W$ and $\mathcal{G}$, a geometric point $x$ of $W$ corresponds to a pair $(\mathcal{H}(x), \mathscr{G}(x))$ where $\mathscr{G}(x)$ is a subalgebra of $\mathcal{H}(x)$ of rank $n$. Let $p: W \rightarrow Z$ be the structure map. Then $\mathcal{H}(x)=\mathscr{G}(p(x))$ has Cartan rank $n$. Let $Y=\{w \in W \mid \mathcal{G}(w)$ is Cartan in $\mathcal{H}(w)\}$. By Corollary $1.11, Y$ is locally closed in $W$. As sets, $Y=\operatorname{Lag}_{n}(G)$, and we give $\operatorname{Lag}_{n}(G)$ the structure of algebraic set coming from this equality. The subalgebra bundles on $\operatorname{Lag}_{n}(G)$ are $\mathscr{K}=\mathcal{H} \mid Y$ and $\mathcal{C}=\mathscr{T} \mid Y$.

We could simultaneously parametrize all left algebraic group structures on $G$ by considering the disjoint union, over all $n$, of $\operatorname{Lag}_{n}(G)$. This moduli space then projects to $\operatorname{Nuc}(G)$. In $\$ 2$ we describe the structure of $\operatorname{Nuc}(G)$ given by Theorem 1.3 , and in $\$ 3$ we consider a section to the projection from left algebraic group structures to $\operatorname{Nuc}(G)$. This is only a morphism over $\operatorname{Nuc}_{n}(G)$, for each $n$. Thus we confine our attention to the moduli spaces $\operatorname{Lag}_{n}(G)$, rather than their union.

2. The space of nuclei. In this section we will explicitly describe the space of nuclei of an FR group as a linear algebraic variety. The basic tool is the following lemma from Lie algebra.

LEMMA 2.1. Let L be a Lie algebra which is a semidirect product of the ideal $K$ and the subalgebra $H$. Then there is a one-one correspondence between ideals of $L$ which are direct sum complements to $\boldsymbol{H}$ and the linear space of linear functions $f: K \rightarrow H$ satisfying

(1) $f([h, k])=[h, f(k)]$ for $h \in H$ and $k \in K$,

(2) $f\left(\left[k_{1}, k_{2}\right]\right)=\left[f\left(k_{2}\right), f\left(k_{1}\right)\right]$ for $k_{1}, k_{2} \in K$. 
Proof. Let $f$ be a linear function satisfying (1) and (2), and let $K_{1}=\{k+f(k), k$ $\in K\}$. It is routine to verify that $K_{1}$ is a direct sum complement to $H$ in $L$. If $k+f(k) \in K_{1}, h \in H$ and $k^{\prime} \in K$, then $[h, k+f(k)]=[h, k]+f([h, k])$ is in $K_{1}$ by (1), and $x=\left[k^{\prime}, k+f(k)\right]$ is in $K$ with $f(x)=f\left(\left[k^{\prime}, k\right]\right)+f\left(\left[k^{\prime}, f(k)\right]\right)$. Apply (2) to the first summand and (1) to the second, so $f(x)=\left[f(k), f\left(k^{\prime}\right)\right]+\left[f\left(k^{\prime}\right), f(k)\right]=$ 0 , and $x=x+f(x) \in K_{1}$. Thus $K_{1}$ is an ideal.

If $K_{1}$ is any ideal of $L$ which is a direct sum complement to $H$, let $K_{0}=\{k \in K \mid$ there is $h \in H$ with $\left.k+h \in K_{1}\right\}$. $K_{0}$ is a subspace of $K$ with $K_{1} \subseteq K_{0}+H$, so $K_{0}=K$. Every element of $K_{1}$ has a unique representation of the form $k+f(k)$, where $f: K \rightarrow H$ is a function, linear because of uniqueness. If $k+f(k) \in K_{1}$ and $h \in H$, then $[h, k+f(k)]=[h, k]+[h, f(k)]$ is in $K_{1}$ with $[h, k]$ in $K$ and $[h, f(k)]$ in $H$, so $f([h, k])=[h, f(k)]$, and $f$ satisfies (1). If $k^{\prime}$ is in $K,\left[k^{\prime}, k+f(k)\right]=\left[k^{\prime}, k\right]$ $+\left[k^{\prime}, f(k)\right]$ is in $K_{1}$ and $K$, so $f\left(\left[k^{\prime}, k+f(k)\right]\right)=f\left(\left[k^{\prime}, k\right]\right)+\left[f\left(k^{\prime}\right), f(k)\right]=0$, and $f$ satisfies (2).

We can use Lemma 2.1 to get a bijection between the set of nuclei and a linear space.

COROLlaRy 2.2. Let $G$ be an $F R$ group, $H$ a maximal reductive subgroup of $G$ and $K$ a nucleus of $G$. Then there is a one-one correspondence between $\operatorname{Nuc}(G)$ and $\operatorname{Hom}_{\mathrm{C}}(L(K) /[L(G), L(K)], \operatorname{Rad}(L(H)))$.

Proof. By Lemmas 1.1 and 2.1, we have a bijection between $\operatorname{Nuc}(G)$ and the linear functions $f: L(K) \rightarrow L(H)$ satisfying Lemma 2.1. But then $f(L(K))$ is an ideal of $L(H)$ by Lemma 2.1, hence contained in $\operatorname{Rad}(L(H))$ (since $L(K)$ is solvable, the second condition of Lemma 2.1 implies $f(L(K))$ is solvable). Since $H$ is reductive, $\operatorname{Rad}(L(H))$ is the center $Z$ of $L(H)$. As $Z$ is abelian, condition (2) of Lemma 2.1 forces $f([L(K), L(K)])=0$, and as $Z$ is central, condition (1) of Lemma 2.1 forces $f([L(H), L(K)])=0$. Thus the linear functions in question are those from $K$ to $Z$ vanishing on $[L(K), L(K)]+[L(H), L(K)]=[L(G), L(K)]$.

Of course, the dimension of the linear space in Corollary 2.2 should be independent of the choice of $K$. To see this, we note that $[L(G), L(G)]=[L(H), L(H)]+$ $[L(G), L(K)]$, and the sum is direct, so the dimension of $[L(G), L(K)]$ is independent of the choice of $K$, as is the dimension of $L(K)$.

We next see that the bijection of Corollary 2.2 is an isomorphism of the algebraic set structure of $\operatorname{Nuc}(G)$ defined in $\$ 1$ with the affine space of linear maps.

THEOREM 2.3. Let $G$ be a FR group, $H$ a maximal reductive subgroup of $G$ and $K a$ nucleus of $G$. Then there is an isomorphism between the algebraic set $\operatorname{Nuc}(G)$ and the linear variety $\operatorname{Hom}_{\mathrm{C}}(L(K) /[L(G), L(K)], \operatorname{Rad}(L(H)))$.

Proof. Let $\mathfrak{g}=L(G), \mathfrak{h}=l(H), \mathfrak{f}=L(K)$ and $r=\operatorname{dim}(K)$. Then $\mathfrak{g}=\mathfrak{f} \times \mathfrak{h}$ as vector spaces, and there is an embedding $p$ from $\operatorname{Hom}_{\mathbb{C}}(\mathfrak{f}, \mathfrak{h})$ to the Grassmann variety $G(r, g)$ of $r$ planes in $g$ given by $p(f)=\{x+f(x) \mid x \in \mathfrak{f}\}$. By Theorem 1.3 there is a subbundle $\mathcal{K}$ of $\mathrm{g} \times \operatorname{Nuc}(G)$ such that $x$ in $\operatorname{Nuc}(G)$ corresponds to the ideal $\mathcal{K}(x)$ of $\mathrm{g}$. The construction of Theorem 1.3 then gives an embedding $q$ : $\operatorname{Nuc}(G) \rightarrow G(r, g)$ which sends $x$ to $\mathscr{K}(x)$. The correspondence of Lemma 2.1 
sends an ideal $K_{1}$ in $\operatorname{Nuc}(G)$ to the linear function $f: \mathfrak{t} \rightarrow \mathfrak{h}$ whose graph is $K_{1}$. If $K_{1}$ is given by the geometric point $x$ of the algebraic set $\operatorname{Nuc}(G)$, then $p(f)=q(x)$. Let $V$ be the subspace of $\operatorname{Hom}_{C}(\mathfrak{f}, \mathfrak{h})$ consisting of linear maps satisfying (1) and (2) of Lemma 2.1. Then by Lemma $2.1, p(V)=q(\operatorname{Nuc}(G))$, so $p^{-1} q$ sends $\operatorname{Nuc}(G)$ isomorphically to $V$. By Corollary $2.2, V$ is isomorphic to $\operatorname{Hom}_{\mathbf{C}}(\mathfrak{f} /[\mathfrak{g}, \mathfrak{f}], \operatorname{Rad}(\mathfrak{h}))$, and composing this isomorphism with $p^{-1} q$ gives the result.

Let $G$ be an FR group whose nuclei have dimension $r$. By Proposition 1.6, $\operatorname{Nuc}_{r}(G)$, the set of nilpotent nuclei of $G$, is a closed subset of $\operatorname{Nuc}(G)$. In [2, Theorem 8.6, p. 135], Hochschild and Mostow show that the set of nilpotent nuclei of $G$ carries the structure of a linear variety. We will now see that the structure on the set of nilpotent nuclei given by Proposition 1.6 and Theorem 2.3 is the same as that discovered by Hochschild and Mostow.

LEMMA 2.4. Let $L$ be a Lie algebra which is the semidirect product of a nilpotent ideal $K$ and a reductive subalgebra $H$ with $K$ a semisimple $H$-module. Then Lemma 2.1 gives a one-one correspondence between the set of nilpotent ideals of $L$ which are direct sum complements to $H$, and $\operatorname{Hom}_{\mathrm{C}}\left(K /[L, K], \operatorname{Rad}(H)^{K}\right)$.

Proof. Let $R=\operatorname{Rad}(H)^{K}$. $K+R$ is a nilpotent ideal of $L$, and hence is contained in the maximal nilpotent ideal $M$ of $L$. Let $K_{1}$ be a nilpotent ideal of $L$ which is a direct sum complement to $H$, and let $f$ be the corresponding function given by Lemma 2.1. As in Corollary $2.2, f(K)$ is contained in $\operatorname{Rad}(H)$. Since $K_{1}$ and $K$ are inside $M$, so is $f(K)$, so it acts nilpotently on $K$. Since $\operatorname{Rad}(H)$ acts semisimply on $K, f(K)$ acts trivially on $K$ so $f(K)$ is inside $R$. If conversely $f$ : $K \rightarrow H$ satisfies (1) and (2) of Lemma (2.1) with $f(K)$ contained in $R$, then the corresponding ideal is contained in $K+R$ so is nilpotent.

TheORem 2.5 [2, Theorem 8.6, p. 125]. Let $G$ be an $F R$ group and $H$ a maximal reductive subgroup. Assume that $G$ has a nilpotent nucleus. Let $\mathrm{m}$ be the maximal nilpotent ideal of $L(G)$, let $\mathfrak{p}=\mathfrak{m} \cap L(H)$ and let $\mathfrak{n}=\operatorname{Rad}([L(G), L(G)])$. Then the set of nilpotent nuclei of $G$ is isomorphic under the isomorphism of Theorem 2.3 with the linear variety $\operatorname{Hom}_{\mathbf{C}}(\mathfrak{m} /(\mathfrak{p}+\mathfrak{n}), \mathfrak{p})$.

Proof. Let $K$ be a nilpotent nucleus of $G$, let $\mathfrak{g}=L(G)$, let $\mathfrak{h}=L(H)$ and let $\mathfrak{f}=L(K)$. Let $\mathfrak{h}_{1}$ be the centralizer of $\mathfrak{f}$ in $\mathfrak{h}$ and let $\mathfrak{h}_{2}$ be the centralizer of $\mathfrak{f}$ in $\operatorname{Rad}(\mathfrak{h})$. Then $\mathfrak{f}+\mathfrak{h}_{1}$ is contained in $\mathfrak{m}$, and $\mathfrak{m}$ is contained in $\operatorname{Rad}(\mathfrak{g})=\mathfrak{f}+\operatorname{Rad}(\mathfrak{h})$. Then $\mathfrak{m}=\mathfrak{f}+\mathfrak{p}$ and $\mathfrak{p}$ is contained in $\operatorname{Rad}(\mathfrak{h})$. If $h$ in $\operatorname{Rad}(\mathfrak{h})$ is nilpotent on $\mathfrak{f}, h$ is in $\mathfrak{h}_{2}$ so $\mathfrak{p}=\mathfrak{h}_{2}$. Now $[\mathfrak{g}, \mathfrak{g}]=[\mathfrak{g}, \mathfrak{t}]+[\mathfrak{h}, \mathfrak{h}]$ and the second summand is semisimple, so $\mathfrak{n}=\operatorname{Rad}[\mathfrak{g}, \mathfrak{g}]=[\mathfrak{g}, \mathfrak{t}]$. Thus $\mathfrak{m} /(\mathfrak{p}+\mathfrak{m})=(\mathfrak{f}+\mathfrak{p}) /(\mathfrak{p}+[\mathfrak{g}, \mathfrak{f}])=\mathfrak{f} /[\mathfrak{g}, \mathfrak{f}]$ so $\operatorname{Hom}_{\mathbf{C}}(\mathfrak{m} /(\mathfrak{p}+\mathfrak{m}), \mathfrak{p})=\operatorname{Hom}_{\mathbf{C}}\left(\mathfrak{f} /[\mathfrak{g}, \mathfrak{t}], \mathfrak{h}_{2}\right)$ and the result follows from Lemma 2.4 and Corollary 2.2 .

If the FR group $G$ has a nilpotent nucleus, $G$ carries an algebraic group structure, and if $G$ has a nonnilpotent nucleus, $G$ carries a left algebraic group structure which is not an algebraic group. Using Theorems 2.3 and 2.5, we can determine when such structures exist. 
THEOREM 2.6. Let $G$ be an algebraic group. Then every left algebraic group structure on $G$ is an algebraic group structure if and only if the radical of $L(G)$ is nilpotent.

Proof. Let $H$ be a maximal reductive subgroup of $G$ and let $U$ be the unipotent radical of $G$. Every left algebraic group structure on $G$ is algebraic if and only if every nucleus of $G$ is nilpotent. Now $U$ is a nilpotent nucleus of $G$, so by Theorem 2.3, Lemma 2.4 and Theorem 2.5, every nucleus of $G$ is nilpotent if and only if $\operatorname{Rad}(L(H))$ centralizes $L(U)$, i.e., if and only if $\operatorname{Rad}(L(G))=L(U)+\operatorname{Rad}(L(H))$ is nilpotent.

In particular, a solvable nonnilpotent algebraic group must always have left algebraic group structures which are not algebraic.

The calculation of the space of nuclei of any given FR group is an elementary matter using Theorem 2.3. As an illustration, we calculate the following example.

EXAMPLE 2.7. Let $\boldsymbol{G}$ be the (algebraic) group $\mathbf{C} \times \mathbf{C} \times \mathbf{C}^{*}$ with operation

$$
(z, x, t)\left(z^{\prime}, x^{\prime}, t^{\prime}\right)=\left(z+z^{\prime}, x+t x^{\prime}, t t^{\prime}\right) \text {. }
$$

For each $a$ in $\mathbf{C}, K_{a}=\{(z, x, \exp (a z)) \mid z, x \in \mathbf{C}\}$ is a nucleus of $G$. These are the only nuclei. $L(G)$ has basis $A, B, C$ with $\exp _{G}(z A)=(z, 0,1), \exp _{G}(x B)=(0, x, 1)$ and $\exp _{G}(u C)=(0,0, \exp (u))$. Then $[A, B]=[A, C]=0$, and $[C, B]=B . H=0$ $\times 0 \times C^{*}$ is a maximal reductive subgroup of $G$ with $L(H)=\operatorname{Rad}(L(H))$ spanned by $C . K_{0}$ is a nucleus of $G$ with $L\left(K_{0}\right)$ spanned by $\{A, B\}$ and $\left[L(G), L\left(K_{0}\right)\right]$ is spanned by $B$. By Theorem $2.3, \operatorname{Nuc}(G)$ is isomorphic to

$$
\operatorname{Hom}_{\mathbf{C}}((\mathbf{C A}+\mathbf{C B}) / \mathrm{CB}, \mathbf{C C})=\operatorname{Hom}_{\mathbf{C}}(\mathbf{C A}, \mathbf{C B}) \text {. }
$$

If $f$ is in this latter space with $f(A)=a C$, the corresponding ideal of $L(G)$ given by Lemma 2.1 is spanned by $\{A+a C, B\}$, so equals $L\left(K_{a}\right)$. Thus the space of nuclei of $G$ is just the complex line. The centralizer of $L\left(K_{0}\right)$ in $L(H)$ is 0 , and so by Lemma 2.4 the unique nilpotent nucleus of $G$ is $K_{0}$.

3. Representatives of left algebraic group structures. In this section we establish the existence of cross section morphisms to the projections $\operatorname{Lag}_{n}(G) \rightarrow \operatorname{Nuc}_{n}(G)$ for an FR group $G$.

We employ the following notations. If $N$ is a nilpotent Lie algebra and $V$ a finite dimensional $N$-module, then $V_{N}$ is the sum of the $N$-root spaces of $V$ corresponding to nonzero roots, and for any subset $S$ of $N, V_{0}(S)=\{x \in V \mid s$ is nilpotent on $x$ for all $s$ in $S\}$. Then $V=V_{0}(N) \oplus V_{N}$ and $N\left(V_{N}\right)=V_{N}$.

The following lemma collects the Lie algebra facts to be used in this section.

LemMA 3.1. Let $L$ be a Lie algebra which is a semidirect product of the ideal $K$ and the subalgebra $H$ which is reductive in $L$. Let $R=\operatorname{Rad}(L), T=\operatorname{Rad}(H)$ and assume $C$ is a Cartan subalgebra of $K$ normalized by $H$. Let $K^{\prime}$ be an ideal of $L$ which is a direct sum complement to $H$. Let $K^{\prime \prime}=\left(K^{\prime}\right)_{0}(C)^{T}$. Then

(1) $K^{\prime}=[R, R]+K^{\prime \prime}$,

(2) $K^{\prime \prime}$ is nilpotent,

(3) $\left(K^{\prime}\right)_{0}\left(K^{\prime \prime}\right)$ is Cartan in $K^{\prime}$. 
Proof. It is easy to check that $K^{\prime}$ is solvable and that $R=K^{\prime}+T=K+T$. Since $[T, C] \subseteq C, K_{0}(C)=C$ and $R=K+T, R_{0}(C)=C+T$. Then $R=K^{\prime}+$ $T$ implies $R_{C}=\left(K^{\prime}\right)_{C}$ and $R_{0}(C)=\left(K^{\prime}\right)_{0}(C)+T$. Since $[T, C] \subseteq C,\left[T,\left(K^{\prime}\right)_{0}(C)\right]$ $\subseteq\left(K^{\prime}\right)_{0}(C)$, so $\left(K^{\prime}\right)_{0}(C)=\left(K^{\prime}\right)_{0}(C)_{T}+K^{\prime \prime}$. Thus $K^{\prime}=\left(K^{\prime}\right)_{C}+\left(K^{\prime}\right)_{0}(C)_{T}+K^{\prime \prime}$. Since $\left[C,\left(K^{\prime}\right)_{C}\right]=\left(K^{\prime}\right)_{C}$ and $\left[T,\left(K^{\prime}\right)_{0}(C)_{T}\right]=\left(K^{\prime}\right)_{0}(C)_{T}$, we have $\left(K^{\prime}\right)_{C}+$ $\left(K^{\prime}\right)_{0}(C)_{T}$ contained in $[R, R]$. Since $R=K^{\prime}+T$ and $T$ is abelian, $[R, R]$ is contained in $K^{\prime}$. So $K^{\prime}=[R, R]+K^{\prime \prime}$ and (1) follows. As $R_{0}(C)=C+T=$ $\left(K^{\prime}\right)_{0}(C)+T, R_{0}(C)^{T}=C^{T}+T=K^{\prime \prime}+T$. Since $C^{T}+T$ is nilpotent, so is $K^{\prime \prime}$, and (2) is obtained. Now $[R, R]$ is a nilpotent ideal of $K^{\prime}$, and $K^{\prime \prime}$ is a nilpotent subalgebra of $K^{\prime}$, so $\left(K^{\prime}\right)_{0}\left(K^{\prime \prime}\right)=[R, R]_{0}\left(K^{\prime \prime}\right)+K^{\prime \prime}$ is a nilpotent self normalizing subalgebra of $K^{\prime}$. Hence it's Cartan, and (3) follows.

COROllary 3.2. Let $G$ be an FR group, $K$ a nucleus of $G$ and $H$ a maximal reductive subgroup of $G$. Suppose $C$ is a Cartan subalgebra of $L(K)$ normalized by $L(H)$. Let $R=\operatorname{Rad}(L(G))$ and let $T=\operatorname{Rad}(L(H))$. Suppose $\operatorname{dim}(C)=t$. Then $q$ : $\operatorname{Nuc}_{t}(G) \rightarrow \operatorname{Lag}_{t}(G)$ given by $q\left(K^{\prime}\right)=\left(K^{\prime},\left(K^{\prime}\right)_{0}\left(\left(K^{\prime}\right)_{0}(C)^{T}\right)\right)$ is a cross section to the projection $\operatorname{Lag}_{t}(G) \rightarrow \operatorname{Nuc}_{t}(G)$.

Proof. Apply Lemma 3.1(3) in Definitions 1.2 and 1.12.

We have not yet shown that $q$ in Corollary 3.2 is a morphism. This requires the following lemma.

LEMMA 3.3. Let $S$ be a variety and let $L$ be a Lie algebra bundle over $S$. Let $V$ be a subalgebra bundle of $L$ which is locally a direct summand of $L$ and let $C$ be $a$ nilpotent subalgebra bundle of $L$ with $[C, V] \subseteq V$. Let $x \in S$ and suppose $r=$ $\operatorname{dim}\left(V(y)_{0}(C(y))\right)$ is constant on a neighborhood of $x$. Then there is a neighborhood $U$ of $x$ and a subalgebra bundle $V_{0}$ of $V \mid U$ which is a direct summand of $L \mid U$ such that $V_{0}(y)=V(y)_{0}(C(y))$ for all $y$ in $U$.

Proof. We may take $S$ to be affine with coordinate ring $R$ and $\operatorname{dim}\left(V(y)_{0}(C(y))\right)$ constant on $S$. Let $m$ be the maximal ideal of $R$ corresonding to $x$. Replace $L, V$, and $C$ by the appropriate finitely generated projective $R$-modules and denote them by the same letters. Let ( $)$ denote reduction $\bmod m$. Choose $s \in C$ such that $(\bar{V})_{0}(\operatorname{ad} \bar{s})=(\bar{V})_{0}(\bar{C})$. Then $\operatorname{char}(\operatorname{ad} \bar{s}, R, X)=X^{r} \bar{g}$, where $g \in$ $R[X]$ and $\bar{g}(0) \neq 0$. We can assume $V$ is a direct summand of $L$ of constant rank. By shrinking $S$ if necessary we can assume $g(0)$ is a unit of $R$. Then $\operatorname{char}(\operatorname{ad}(s), R, X)=X^{r} g$ and by Remark $1.5 V_{0}(\operatorname{ad} s)$ is a direct summand of $V$, hence $L$, of constant rank $r$. For any maximal ideal $n$ of $R,(V / n V)_{0}(C / n C)$ is contained in $V_{0}(\mathrm{ad} s) / n V_{0}(\mathrm{ad} s)$ and both have dimension $r$, so they are equal. Thus $V_{0}=V_{0}(\mathrm{ad} s)$ gives the desired subalgebra.

Proposition 3.4. The map of Corollary 3.2 is a morphism.

Proof. We continue with the notation of Corollary 3.2. Let $S=\mathrm{Nuc}_{t}(G)$, let $\mathfrak{g}=L(G)$ and let $\mathcal{K} \subseteq \mathfrak{g} \times S$ be the bundle of Corollary 1.8. For each $x$ in $S$, $R_{0}(C)=\mathscr{K}(x)_{0}(C) \oplus T$, so $\operatorname{dim}\left(\mathcal{K}(x)_{0}(C)\right)$ is constant. Now $C \times X$ is a nilpotent subalgebra bundle of $g \times S$ with $(C \times S)(x)=C$ for all $x$ in $S$ and $[C \times S, \mathcal{K}$ ] $\subseteq \mathcal{K}$. Fix $x \in S$. By Lemma 3.3 there is a neighborhood $S^{\prime}$ of $x$ and a subalgebra 
bundle $\mathscr{K}_{0}$ of $\mathscr{K} \mid S^{\prime}$ with $\mathscr{K}_{0}(y)=\mathscr{K}(y)_{0}(C)$ for all $y$ in $S^{\prime}$, and such that $\mathscr{K}_{0}$ is a direct summand of $L \times S^{\prime}$. Since $R_{0}(C)^{T}=\mathscr{K}(y)_{0}(C)^{T} \oplus T=\mathscr{K}_{0}(y)_{0}(T) \oplus T$ for each $y$ in $S^{\prime}, \operatorname{dim}\left(\mathcal{K}_{0}(y)_{0}(T)\right)$ is constant. $T \times S^{\prime}$ is a nilpotent subalgebra bundle of $L \times S^{\prime}$ normalizing $\mathscr{K}_{0}$, so by Lemma 3.3 there is a neighborhood $S^{\prime \prime}$ of $x$ in $S^{\prime}$ and a subalgebra bundle $\mathcal{K}^{\prime \prime}$ of $\mathcal{K}_{0} \mid S^{\prime \prime}$ with $\mathcal{K}^{\prime \prime}(y)=\mathcal{K}_{0}(y)_{0}(T)=\mathcal{K}_{0}(y)^{T}$ for each $y$ in $S^{\prime \prime}$ with $\mathcal{K}^{\prime \prime}$ a direct summand of $L \times S^{\prime \prime}$. By Lemma 3.1(2), $\mathcal{K}^{\prime \prime}(y)$ is nilpotent for each $y$ in $S^{\prime \prime}$, so by Lemma $1.9 \mathcal{K}^{\prime \prime}$ is nilpotent. By Lemma 3.1(3), $\left(\mathscr{K} \mid S^{\prime \prime}\right)(y)_{0}\left(\mathcal{K}^{\prime \prime}(y)\right)$ is Cartan in $\left(\mathcal{K} \mid S^{\prime \prime}\right)(y)$ for each $y$ in $S^{\prime \prime}$, so it has dimension $t$. By Theorem 2.3, there is a neighborhood $U$ of $x$ in $S^{\prime \prime}$ and a subalgebra bundle $\mathscr{D}$ of $\mathscr{K} \mid U$ such that $\mathscr{D}(y)=\left(\mathscr{K} \mid S^{\prime \prime}\right)(y)_{0}\left(\mathscr{K}^{\prime \prime}(y)\right)$ for each $y$ in $U$. Now $\mathscr{D}$ is a Lie subalgebra bundle of $\mathscr{K} \mid U$ of constant rank $t$, so by the universal property of $\operatorname{Lie}_{\mathscr{K} / s}^{t}$ [1, Theorem 2.2, p. 12], $\mathscr{D}$ defines a morphism $f: U \rightarrow \mathrm{Lie}_{\mathscr{K} / s}^{t}$ such that $f(y)=(\mathcal{K}(y), \mathscr{D}(y))$. By construction, $f(y)=q(y)$ for $y \in U$, so $q$ is locally a morphism, and hence a morphism.

Now suppose $G$ is an FR group with $g=L(G)$, and let $G$ act on $g$ via the adjoint representation. If $\mathfrak{t}$ is the Lie algebra of a nucleus of $G$ then $G$ stabilizes $\mathfrak{f}$ under the adjoint action, and acts transitively on the Cartan subalgebras of $\mathfrak{t}$. Thus $G$ acts on $\operatorname{Lag}_{t}(G)$ by $g(\mathfrak{f}, \mathfrak{c})=(\mathfrak{f}, \operatorname{Ad}(g)(\mathfrak{c}))$ and the projection $p: \operatorname{Lag}_{t}(G) \rightarrow$ $\operatorname{Nuc}_{t}(G)$ identifies $\mathrm{Nuc}_{t}(G)$ with the space of $G$-orbits on $\operatorname{Lag}_{t}(G)$. Thus the maps $q$ of Corollary 3.2, for all $t$, give a one-one correspondence between $G$-conjugacy classes of left algebraic group structures on $G$ and the affine space $\operatorname{Nuc}(G)$. If $H$ is a maximal reductive subgroup of $G$, then $\operatorname{Nuc}(G)$ is isomorphic to $\operatorname{Hom}_{C}(\mathfrak{f} /[\mathfrak{g}, \mathfrak{f}], \operatorname{Rad}(L(H)))$ by Theorem 2.3. In [9, Theorem 3.2, p. 251], Hochschild and Mostow find a mapping of an affine space $V=$ $\operatorname{Hom}\left(\operatorname{Hom}\left(T, C^{*}\right), \operatorname{Hom}(G, C)\right)$, where $T$ is a maximal torus in $G$, the outside "Hom" is group homomorphism and the inside ones are analytic group homomorphisms, to the set of left algebraic group structures on $G$ such that the image meets every $G$-conjugacy class. Now $\operatorname{Hom}(G, \mathbf{C})=\operatorname{Hom}_{\mathbf{C}}(\mathfrak{f} /[\mathfrak{g}, \mathfrak{f}], \mathbf{C})$, so $\operatorname{dim}(V)=$ $\operatorname{dim}(T) \cdot \operatorname{dim}(\mathfrak{f} /[\mathfrak{g}, \mathfrak{f}])$. If $H$ is abelian (i.e., if $G$ is solvable), $\operatorname{dim} T=$ $\operatorname{dim}(\operatorname{Rad}(L(H)))$, so $V$ and $\operatorname{Nuc}(G)$ have the same direction, but in all other cases $\operatorname{dim}(V)$ is larger.

\section{REFERENCES}

1. W. Haboush, The scheme of Lie subalgebras of a Lie algebra and the equivariant cotangent map, Nagoya Math. J. 53 (1974), 59-70.

2. G. Hochschild and G. Mostow, On the algebra of representative functions of an analytic group, Amer. J. Math. 83 (1961), 111-136.

3. __ Affine embeddings of complex analytic homogeneous spaces, Amer. J. Math. 87 (1965), 807-839.

4. I. Kaplansky, Lie algebras and locally compact groups, Univ. of Chicago Press, Chicago, Ill., 1971.

5. M.-A. Krus and M. Ojanguren, Théorie de la descente et algèbres d'Azumaya, Lecture Notes in Math., vol. 389, Springer-Verlag, Berlin and New York, 1974.

6. A. Magid, Left algebraic groups, J. Algebra 35 (1975), 253-272.

7. __ Analytic left algebraic groups, Amer. J. Math. 99 (1977), 1045-1059.

8. __ Analytic left algebraic groups. II, Trans. Amer. Math. Soc. 238 (1978), 165-177.

9. G. Hochschild and G. Mostow, Deformations of affine embeddings of complex analytic homogeneous spaces, Amer. J. Math. 88 (1966), 244-257.

Department of Mathematics, University of OKLahoma, Norman, OKLAhoma 73019 